\title{
Katzenallergene: Aussperren allein genügt nicht
}

\section{In einer britischen Studie wurde das Katzenhaarallergen Fel d 1 regelmäßig in Wohnungen von Nicht-Katzenbesitzern gefunden. Neben dem Hausstaub war häufig auch die Raumluft belastet.}

$\mathrm{n}$
$\mathrm{de}$
$\mathrm{de}$ n Großbritannien gehören Katzen zu den beliebtesten Haustieren. In jedem vierten Haushalt lebt wenigstens ein Exemplar, Mehrkatzenhaushalte sind keine Seltenheit. Die Arbeitsgruppe um A. Custovic et al. vom Wythenshawe Hospital in Manchester analysierte vor diesem Hintergrund in 125 Wohnungen der britischen Industriestadt die Menge an Katzenallergenen in Hausstaub und Raumluft. Dabei handelte es sich in 50 Fällen um Wohnungen von Katzenbesitzern, 75 der (Kontroll-)Wohnungen waren katzenfrei.

Zunächst zu den Katzenbesitzern: Jede Katze produziert pro Tag 3 bis 7 $\mu \mathrm{g}$ des Antigens Fel d 1. Da sich Katzen am liebsten auf Sesseln und Sofas. tummeln, durften die Wissenschaftler in den Staubproben von Polstermöbeln die höchsten Allergen-Konzentrationen erwarten. Sie fanden im Durchschnitt $380 \mu \mathrm{g} / \mathrm{g}$ pro Gramm Staub (Höchswert: $6000 \mu \mathrm{g}$ ). Im Wohnzimmerteppich war die Konzentration mit $237 \mu \mathrm{g}$ Fel d 1 pro Gramm schon deutlich niedriger. Da die Katzen sich seltener im Schlafzimmer aufhielten, lag die Konzentration in Schlafzimmerteppich und Matratze noch einmal um den Faktor 4,3 niedriger $(55 \mu \mathrm{g}$ Fel d 1 pro Gramm).

Die Allergenmengen liegen im Rahmen der Werte aus früheren Untersuchungen anderer Autoren. Neu ist, daß Custovic et al. Katzenallergene regelmäßig auch in Wohnungen nachweisen konnten, in denen gar keine Katzen gehalten wurden. Fel d 1 war in den Staubproben aller 75 untersuchten Wohnungen von Nicht-Katzenbesitzern vorhanden. Die höchsten Allergenmengen wurden wiederum auf den Polstermöbeln gemessen. Die Konzentration war zwar 314fach geringer als auf den Wohnmöbeln von Katzenbesitzern. Doch Mengen von bis zu 61,9 $\mu \mathrm{g}$ Fel d 1 pro Gramm können durchaus ausreichen, bei Allergikern eine Exazerbation der Erkrankung auszulösen.

Offenbar läßt sich auch bei NichtKatzenbesitzern keine völlige Elimination der Allergene erreichen. Die Autoren vermuten, daß es über die Kleidung von Katzenbesitzern in der Bekanntschaft zu einem ,,passiven Transfer" auf die Polstermöbel in katzenfreien Zonen kommt. In Manchester scheint die Verbreitung von Katzen jedenfalls hoch genug zu sein, um über diesen Mechanismus zu einer ubiquitären Verbreitung von Fel d 1 $\mathrm{zu}$ führen. In früheren Untersuchungen hatten die Autoren Katzenallergene regelmäßig auch in Krankenhäusern, Kinos und öffentlichen Gebäuden aufgespürt.

Dennoch ist eine Sanierung der Wohnung häufig unumgänglich, vor allem, wenn Katzenallergene Asthmaanfälle auslösen. Bis zu 40\% der Kinder mit Asthma sind gegen Fel d 1 sensibilisiert. Ihr Risiko ist erheblich: Denn auch in der Raumluft fanden sich erhebliche Mengen an Katzenallergen: Custovic konnte das Allergen in der Raumluft aller Haushalte mit Katzen nachweisen. Die Konzentration lag zwischen 0,7 und $38 \mathrm{ng}$ Fel d $1 / \mathrm{m}^{3}$ Luft. Auch in 22 von 75 Wohnungen ohne Katzen wurde eine aerogene Allergenbelastung gemessen (Konzentration 0,24 bis $\left.1,78 \mathrm{ng} / \mathrm{m}^{3}\right)$. Sicher vor Katzen- allergenen sind Asthmatiker nur im Schlafzimmer ihrer katzenfreien Wohnung. Fel d 1 war hier in keiner von 20 Proben nachweisbar.

Nach dem Aussperren der Katze nimmt die Allergen-Konzentration ab. In einem Haushalt ging die Luftbelastung zwei Tage nach Exilierung von vier Hauskatzen um 61,7\% zurück. Eine Analyse der Partikelgröße ergab aber, daß dies vor allem auf eine Reduktion der an große Partikel (> 4,8 $\mu \mathrm{m})$ gebundenen Allergene zurückzuführen war. Sie fielen auf $13 \%$ des Ausgangswertes. Die Menge des an kleine Luftpartikel gebundenen Fel d 1 nahm erst ab dem 4. Tag und danach nur langsam ab. Nach 14 Tagen war eine Reduk-

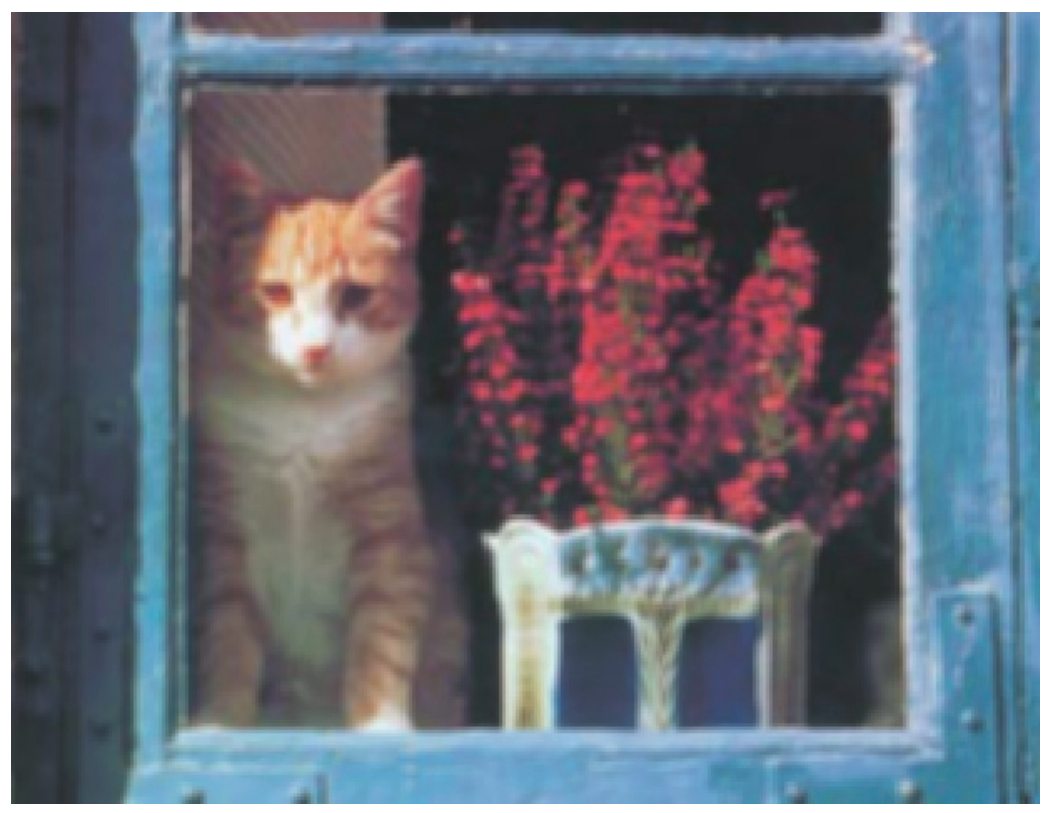

Geschützt hinter der Fensterscheibe?

(Abb.: Bayer USA Annual Report 1988)

tion um $67 \%$ erreicht. Da vor allem die kleinen Partikel wegen ihres tieferen Eindringens in die Atemwege für die Asthmaanfälle verantwortlich sind, kann den Patienten der Besuch von Wohnungen, die zu seiner Schonung kurzfristig katzenfrei gehalten werden, nicht empfohlen werden. Die Autoren vermuten, daß es Monate dauert, bis eine Wohnung als weitgehend katzenallergenfrei erklärt werden kann. (rme)

Quelle: A. Custovic et al.: Distribution, aerodynamic characQuelle: A. Custovic et al.: Distribution, aerodynamic charactish homes. Thorax (1998); 53:33-38. 